\title{
Some Inclusion Relations Associated with Generalized Fractional Differintegral Operator
}

\author{
Amit Soni $^{1}$ and Shashi Kant ${ }^{2}$ \\ ${ }^{1}$ Department of Mathematics, Government Engineering College, Bikaner 334001, India \\ ${ }^{2}$ Department of Mathematics, Government Dungar College, Bikaner 334001, India
}

Correspondence should be addressed to Amit Soni; aamitt81@gmail.com and Shashi Kant; drskant.2007@yahoo.com

Received 15 November 2012; Revised 25 January 2013; Accepted 30 January 2013

Academic Editor: Ahmed Zayed

Copyright (C) 2013 A. Soni and S. Kant. This is an open access article distributed under the Creative Commons Attribution License, which permits unrestricted use, distribution, and reproduction in any medium, provided the original work is properly cited.

A generalized fractional differintegral operator is used to define some new subclasses of analytic functions in the open unit disk $\mathbb{U}$. For each of these new function classes, several inclusion relationships are established.

\section{Introduction and Definitions}

Let $\mathbb{A}$ be the class of normalized functions $f$ of the form

$$
f(z)=z+\sum_{n=2}^{\infty} a_{n} z^{n}
$$

which are analytic in the open unit disk $\mathbb{U}=\{z \in D,|z|<1\}$. If $f \in \mathbb{A}$ is given by (1) and $g \in \mathbb{A}$ given by $g(z)=$ $z+\sum_{n=2}^{\infty} b_{n} z^{n}$ in $z \in \mathbb{U}$, then the Hadamard product (or convolution) of $f$ and $g$ is defined by

$$
(f * g)(z)=z+\sum_{n=2}^{\infty} a_{n} b_{n} z^{n}
$$

Let $P_{k}(\alpha)$ denote the class of functions $h(z)$ analytic in the unit disk $\mathbb{U}$, satisfying the properties $h(0)=1$ and

$$
\int_{0}^{2 \pi}\left|\mathbb{R}\left(\frac{h(z)-\alpha}{1-\alpha}\right)\right| d \theta \leq k \pi \quad\left(z=r e^{i \theta} ; 0 \leq \alpha<1 ; k \geq 2\right) .
$$

This class $P_{k}(\alpha)$ has been introduced in [1]. Note that, for $\alpha=$ 0 , we obtain the class $P_{k}$ defined and studied in [2], and for $k=$ 2 , we have the class $P(\alpha)$ of functions with positive real part greater than $\alpha$. In particular, $P(0)$ is the class $P$ of functions with positive real part. From (3), we can easily deduce that $h \in P_{k}(\alpha)$ if and only if

$$
h(z)=\left(\frac{k}{4}+\frac{1}{2}\right) h_{1}(z)-\left(\frac{k}{4}-\frac{1}{2}\right) h_{2}(z), \quad h_{1}, h_{2} \in P(\alpha) .
$$

Following the recent investigations [3] (see also $[4,5]$ ), we have the following subclasses:

$$
\begin{gathered}
R_{k}(\alpha)=\left\{f \in A: \frac{z f^{\prime}(z)}{f(z)} \in P_{k}(\alpha), z \in \mathbb{U}\right\}, \\
V_{k}(\alpha)=\left\{f \in A: \frac{\left(z f^{\prime}(z)\right)^{\prime}}{f^{\prime}(z)} \in P_{k}(\alpha), z \in \mathbb{U}\right\}, \\
P_{k}^{\prime}(\alpha)=\left\{f \in A: f^{\prime}(z) \in P_{k}(\alpha), z \in \mathbb{U}\right\}, \\
T_{k}(\beta, \alpha)=\left\{f \in A: g \in R_{2}(\alpha), \frac{z f^{\prime}(z)}{g(z)} \in P_{k}(\beta), z \in \mathbb{U}\right\}, \\
=\left\{f \in A: g \in V_{2}(\alpha), \frac{\left(z f^{\prime}(z)\right)^{\prime}}{g^{\prime}(z)} \in P_{k}(\beta), z \in \mathbb{U}\right\} .
\end{gathered}
$$


We note that the class $R_{2}(\alpha)=S^{*}(\alpha)$ and $V_{2}(\alpha)=\mathscr{K}(\alpha)$ are, respectively, the subclasses of $\mathbb{A}$, consisting of functions which are starlike of order $\alpha$ and convex of order $\alpha$ in $\mathbb{U}$. The class $T_{2}^{*}(\beta, \alpha)=C^{*}(\beta, \alpha)$ was considered by Noor [6], and $T_{2}^{*}(0,0)=C^{*}$ is the class of quasiconvex univalent functions which was first introduced and studied in [7]. It can be easily seen from the above definition that

$$
\begin{gathered}
f(z) \in V_{k}(\alpha) \Longleftrightarrow z f^{\prime}(z) \in R_{k}(\alpha), \\
f(z) \in T_{k}^{*}(\beta, \alpha) \Longleftrightarrow z f^{\prime}(z) \in T_{k}(\beta, \alpha) .
\end{gathered}
$$

We recall here the following family of generalized fractional integral operators due to Srivastava et al. [8] and generalized fractional derivative operators due to Raina and Nahar [9] (see also $[10,11]$ ).

Definition 1. Let $\lambda>0$ and $\mu, \eta \in \mathbb{R}$. Then the fractional integral operator $\mathscr{F}_{0, z}^{\lambda, \mu, \eta}$ is defined by

$$
\begin{aligned}
& \mathscr{I}_{0, z}^{\lambda, \mu, \eta} f(z) \\
& \quad=\frac{z^{-\lambda-\mu}}{\Gamma(\lambda)} \int_{0}^{z}(z-\zeta)_{2}^{\lambda-1} F_{1}\left(\lambda+\mu,-\eta ; \lambda ; 1-\frac{\zeta}{z}\right) f(\zeta) d \zeta
\end{aligned}
$$

where the function $f(z)$ is analytic in a simply connected region of the complex $z$-plane containing the origin, with the order

$$
f(z)=O\left(|z|^{\varepsilon}\right) \quad(z \longrightarrow 0 ; \varepsilon>\max \{0, \mu-\eta\}-1),
$$

and the multiplicity $(z-\zeta)^{\lambda-1}$ is removed by requiring $\log (z-$ $\zeta)$ to be real when $z-\zeta>0$.

Definition 2. For $m-1 \leqq \lambda<m$ and $\mu, \eta \in \mathbb{R}$ and $m \in \mathbb{N}$, the fractional derivative operator $\mathcal{J}_{0, z}^{\lambda, \mu, \eta}$ is defined by

$$
\begin{aligned}
& \mathscr{J}_{0, z}^{\lambda, \mu, \eta} f(z) \\
&=\frac{d^{m}}{d z^{m}}\left(\frac{z^{\lambda-\mu}}{\Gamma(m-\lambda)}\right. \\
& \times \int_{0}^{z}(z-\zeta)^{m-\lambda-1}{ }_{2} F_{1}(\mu-\lambda, m-\eta ; m-\lambda ; \\
&\left.\left.1-\frac{\zeta}{z}\right) f(\zeta) d \zeta\right),
\end{aligned}
$$

where the multiplicity $(z-\zeta)^{m-\lambda}$ is removed as in the above definition.

The operators $\mathscr{I}_{0, z}^{\lambda, \mu, \eta}$ and $\mathscr{F}_{0, z}^{\lambda, \mu, \eta}$ include the RiemannLiouville and Erdelyi-Kober operators of fractional calculus (see, e.g., [10]). Using the hypotheses of Definitions 1 and 2 the generalized fractional differintegral operator

$$
\mathcal{S}_{0, z}^{\lambda, \mu, \eta}: \mathbb{A} \longrightarrow \mathbb{A}
$$

is defined by (see also [12-15])

$$
\mathcal{S}_{0, z}^{\lambda, \mu, \eta} f(z)=\left\{\begin{array}{r}
\frac{\Gamma(2-\mu) \Gamma(2+\eta-\lambda)}{\Gamma(2+\eta-\mu)} z^{\mu} \mathcal{J}_{0, z}^{\lambda, \mu, \eta} f(z), \\
\frac{\Gamma(2-\mu) \Gamma(2+\eta-\lambda)}{\Gamma(2+\eta-\mu)} z^{\mu} \mathcal{F}_{0, z}^{-\lambda, \mu, \eta} f(z), \\
(\lambda<0),
\end{array}\right.
$$

$(z \in \mathbb{U}, \mu, \eta \in \mathbb{R}, \min \{-\mu, \eta-\lambda, \eta-\mu\}>-2)$.

It is easily seen from (12) that for a function $f$ of the form (1), we have

$$
\begin{aligned}
\mathcal{S}_{0, z}^{\lambda, \mu, \eta} f(z) & \\
= & z+\frac{\Gamma(2-\mu) \Gamma(2+\eta-\lambda)}{\Gamma(2+\eta-\mu)} \\
& \times \sum_{n=2}^{\infty} \frac{\Gamma(n+1) \Gamma(n+\eta-\mu+1)}{\Gamma(n-\mu+1) \Gamma(n+\eta-\lambda+1)} a_{n} z^{n} \\
= & z_{3} F_{2}(1,2,2+\eta-\mu ; 2-\mu, 2+\eta-\lambda ; z) * f(z) .
\end{aligned}
$$

We note that the operator $\mathcal{S}_{0, z}^{\lambda, \mu, \eta}$ is a generalization of many other operators, for example,

$$
\begin{aligned}
& \text { (i) } \begin{aligned}
\mathcal{S}_{0, z}^{0,0,0} f(z) & =f(z), \\
\text { (ii) } \mathcal{S}_{0, z}^{1,1,1} f(z) & =\mathcal{S}_{0, z}^{1,0,0} f(z)=z f^{\prime}(z), \\
\text { (iii) } \mathcal{S}_{0, z}^{2,1,1} f(z)= & z f^{\prime}(z)+z^{2} f^{\prime \prime}(z), \\
\text { (iv) } \mathcal{S}_{0, z}^{\lambda, \lambda, \eta} f(z)= & \mathcal{S}_{0, z}^{\lambda, \mu, 0} f(z), \\
\text { (v) } \mathcal{S}_{0, z}^{-\lambda, 0, \alpha-1} f(z) & =\Omega_{\alpha}^{\lambda} f(z) \\
& \left(\begin{array}{c}
\lambda+\alpha \\
\alpha
\end{array}\right) \frac{\lambda}{z^{\alpha}} \\
& \times \int_{0}^{z} \zeta^{\alpha-1}\left(1-\frac{\zeta}{z}\right)^{\lambda-1} f(\zeta) d \zeta,
\end{aligned}
\end{aligned}
$$

which is a multiplier transformation operator $\Omega_{\alpha}^{\lambda}$ studied by Jung et al. [16],

$$
\text { (vi) } \begin{aligned}
\mathcal{S}_{0, z}^{-1,0, \alpha-1} f(z) & =\mathscr{I}_{\alpha} f(z) \\
& =\frac{\alpha+1}{z^{\alpha}} \int_{0}^{z} \zeta^{\alpha-1} f(\zeta) d \zeta \quad(\alpha>-1)
\end{aligned}
$$

which is the generalized Bernardi-Libra-Livingston integral operator. It can easily be verified from (12) that

$$
\begin{aligned}
& z\left(\mathcal{\delta}_{0, z}^{\lambda, \mu, \eta} f(z)\right)^{\prime} \\
& \quad=(\eta-\lambda+1) \delta_{0, z}^{\lambda+1, \mu, \eta} f(z)-(\eta-\lambda) \delta_{0, z}^{\lambda, \mu, \eta} f(z) .
\end{aligned}
$$


For the generalized fractional differintegral operator $\mathcal{S}_{0, z}^{\lambda, \mu, \eta}$, we now define the following subclasses of $A$.

Definition 3. Let $f(z) \in \mathbb{A}$. Then $f(z) \in R^{\lambda, \mu, \eta}(k, \alpha)$ if and only if $\mathcal{S}_{0, z}^{\lambda, \mu, \eta} f(z) \in R_{k}(\alpha)$, for $z \in \mathbb{U}$.

Definition 4. Let $f(z) \in \mathbb{A}$. Then $f(z) \in V^{\lambda, \mu, \eta}(k, \alpha)$ if and only if $\mathcal{S}_{0, z}^{\lambda, \mu, \eta} f(z) \in V_{k}(\alpha)$, for $z \in \mathbb{U}$.

Definition 5. Let $f(z) \in \mathbb{A}$. Then $f(z) \in T^{\lambda, \mu, \eta}(k, \beta, \alpha)$ if and only if $\mathcal{S}_{0, z}^{\lambda, \mu, \eta} f(z) \in T_{k}(\beta, \alpha)$, for $z \in \mathbb{U}$.

Definition 6. Let $f(z) \in \mathbb{A}$. Then $f(z) \in T_{*}^{\lambda, \mu, \eta}(k, \beta, \alpha)$ if and only if $\mathcal{S}_{0, z}^{\lambda, \mu, \eta} f(z) \in T_{k}^{*}(\beta, \alpha)$, for $z \in \mathbb{U}$.

Definition 7. Let $f(z) \in \mathbb{A}$. Then $f(z) \in P_{k}^{\prime}(\lambda, \mu, \eta, \alpha)$ if and only if $\mathcal{S}_{0, z}^{\lambda, \mu, \eta} f(z) \in P_{k}^{\prime}(\alpha)$, for $z \in \mathbb{U}$.

In this paper we establish some inclusion relationships and some other interesting properties for these subclasses.

\section{Main Inclusion Relationships}

We recall first the following necessary lemma.

Lemma 8 (see [17]). Let $u=u_{1}+i u_{2}$ and $v=v_{1}+i v_{2}$, and let $\phi(u, v)$ be a complex-valued function satisfying the conditions:

(i) $\phi(u, v)$ is continuous in $D \subset \mathbb{C}^{2}$,

(ii) $(1,0) \in D$ and $\mathbb{R} \phi(1,0)>0$,

(iii) $\mathbb{R} \phi\left(i u_{2}, v_{1}\right) \leq 0$ whenever $\left(i u_{2}, v_{1}\right) \in D$ and $v_{1} \leq$ $-(1 / 2)\left(1+u_{2}^{2}\right)$

If $h(z)=1+\sum_{n=2}^{\infty} c_{n} z^{n}$ is a function analytic in $\mathbb{U}$ such that $\left(h(z), z h^{\prime}(z)\right) \in D$ and $\mathbb{R e}\left(\phi\left(h(z), z h^{\prime}(z)\right)\right)>0$, for $z \in \mathbb{U}$, then $\mathbb{R e}(h(z))>0$ for $z \in \mathbb{U}$.

Our first main inclusion relationship is given by the theorem below.

Theorem 9. Let $f \in \mathbb{A}, 0 \leq \alpha<1, \eta-\lambda>1 / 8$, and $\min \{-\mu, \eta-\mu\}>-2$; then

$$
R^{\lambda+1, \mu, \eta}(k, \alpha) \subset R^{\lambda, \mu, \eta}\left(k, \alpha_{1}\right),
$$

where

$$
\begin{aligned}
\alpha_{1}=2 & (2 \alpha \eta-2 \alpha \lambda+1) \\
& \times((2(\eta-\lambda-\alpha)+1) \\
& \left.+\sqrt{4(\eta-\lambda+\alpha)^{2}+4(\eta-\lambda-\alpha)+9}\right)^{-1}
\end{aligned}
$$

and $0 \leq \alpha<\alpha_{1}<1$.
Proof. Let $f \in R^{\lambda+1, \mu, \eta}(k, \alpha)$. Then upon setting

$$
\begin{aligned}
\frac{z\left(\mathcal{S}_{0, z}^{\lambda, \mu, \eta} f(z)\right)^{\prime}}{\mathcal{S}_{0, z}^{\lambda, \mu, \eta} f(z)} & =p(z) \\
& =\left(\frac{k}{4}+\frac{1}{2}\right) p_{1}(z)-\left(\frac{k}{4}-\frac{1}{2}\right) p_{2}(z) \\
& (z \in \mathbb{U}),
\end{aligned}
$$

we see that the function $p(z)$ is analytic in $\mathbb{U}$, with $p(0)=1$ in $z \in \mathbb{U}$. Using the identity (16) in (19) and differentiating with respect to $z$, we get

$$
\begin{aligned}
& \frac{z\left(\mathcal{S}_{0, z}^{\lambda+1, \mu, \eta} f(z)\right)^{\prime}}{\mathcal{S}_{0, z}^{\lambda+1, \mu, \eta} f(z)} \\
& \quad=\left(p(z)+\frac{z p^{\prime}(z)}{p(z)+\eta-\lambda}\right) \in P_{k}(\alpha) \quad(z \in \mathbb{U}) .
\end{aligned}
$$

Let

$$
\phi(z)=\sum_{j=1}^{\infty} \frac{\eta-\lambda+j}{\eta-\lambda+1} z^{j} .
$$

Then, by convolution technique, (see [18]), we have

$$
\begin{aligned}
p(z) * \frac{\phi(z)}{z}= & p(z)+\frac{z p^{\prime}(z)}{p(z)+\eta-\lambda} \\
= & \left(\frac{k}{4}+\frac{1}{2}\right)\left(p_{1}(z) * \frac{\phi(z)}{z}\right) \\
& -\left(\frac{k}{4}-\frac{1}{2}\right)\left(p_{2}(z) * \frac{\phi(z)}{z}\right)
\end{aligned}
$$

and this implies that

$$
\left(p_{i}(z)+\frac{z p_{i}^{\prime}(z)}{p_{i}(z)+\eta-\lambda}\right) \in P(\alpha) \quad(z \in \mathbb{U}, i=1,2) .
$$

We want to show that $p_{i}(z) \in P\left(\alpha_{1}\right)$, where $\alpha_{1}$ is given by (18), and this will show that $p(z) \in P_{k}\left(\alpha_{1}\right)$ for $z \in \mathbb{U}$. Let

$$
p_{i}(z)=\left(1-\alpha_{1}\right) h_{i}(z)+\alpha_{1} \quad(z \in \mathbb{U}, i=1,2) .
$$

Then in view of (23) and (24), we obtain, for $z \in \mathbb{U}, i=1,2$

$$
\begin{aligned}
& \mathbb{R}\left(\left(1-\alpha_{1}\right) h_{i}(z)+\left(\alpha_{1}-\alpha\right)+\frac{\left(1-\alpha_{1}\right) z h_{i}^{\prime}(z)}{\left(1-\alpha_{1}\right) h_{i}(z)+\alpha_{1}+\eta-\lambda}\right) \\
& \quad>0 .
\end{aligned}
$$

We now form a function $\phi(u, v)$ by choosing $u=h_{i}(z)$ and $v=z h_{i}^{\prime}(z)$ in (25). Thus

$$
\phi(u, v)=\left(1-\alpha_{1}\right) u+\alpha_{1}-\alpha+\frac{\left(1-\alpha_{1}\right) v}{\left(1-\alpha_{1}\right) u+\alpha_{1}+\eta-\lambda} .
$$


We can easily see that the first two conditions of Lemma 8 are easily satisfied as $\phi(u, v)$ is continuous in $D=\mathbb{C}-\left(-\left(\alpha_{1}+\eta-\right.\right.$ $\left.\lambda) /\left(1-\alpha_{1}\right)\right) \times \mathbb{C},(1,0) \in D$, and $\mathbb{R}\{\phi(1,0)\}>0$. Now for $v_{1} \leq-(1 / 2)\left(1+u_{2}^{2}\right)$ we obtain

$$
\begin{aligned}
\mathbb{R} & \left\{\phi\left(i u_{2}, v_{1}\right)\right\} \\
& =\mathbb{R}\left(\left(1-\alpha_{1}\right) i u_{2}+\alpha_{1}-\alpha+\frac{\left(1-\alpha_{1}\right) v_{1}}{\left(1-\alpha_{1}\right) i u_{2}+\alpha_{1}+\eta-\lambda}\right) \\
& =\alpha_{1}-\alpha+\frac{\left(1-\alpha_{1}\right) v_{1}\left\{\alpha_{1}+\eta-\lambda\right\}}{\left(\alpha_{1}+\eta-\lambda\right)^{2}+\left(1-\alpha_{1}\right)^{2} u_{2}^{2}} \\
& \leq \alpha_{1}-\alpha-\frac{1}{2} \frac{\left(1-\alpha_{1}\right)\left(\alpha_{1}+\eta-\lambda\right)\left(1+u_{2}^{2}\right)}{\left(\alpha_{1}+\eta-\lambda\right)^{2}+\left(1-\alpha_{1}\right)^{2} u_{2}^{2}} \\
& =\frac{A+B u_{2}^{2}}{2 C},
\end{aligned}
$$

where $A=\left(\alpha_{1}+\eta-\lambda\right)\left\{2\left(\alpha_{1}-\alpha\right)\left(\alpha_{1}+\eta-\lambda\right)-\left(1-\alpha_{1}\right)\right\}, B=$ $\left(1-\alpha_{1}\right)\left\{2\left(\alpha_{1}-\alpha\right)\left(1-\alpha_{1}\right)-\left(\alpha_{1}+\eta-\lambda\right)\right\}$, and $C=\left(\alpha_{1}+\eta-\lambda\right)^{2}+$ $\left(1-\alpha_{1}\right)^{2} u_{2}^{2}>0$.

We note that $\mathbb{R}\left\{\phi\left(i u_{2}, v_{1}\right\} \leq 0\right.$ if and only if $A \leq 0$ and $B \leq 0$. From $A \leq 0$, we obtain $\alpha_{1}$ as given by (18), and $B \leq 0$ gives us $0 \leq \alpha_{1}<1$. This completes the proof.

Theorem 10. Let $f \in \mathbb{A}, 0 \leq \alpha<1, \eta-\lambda>1 / 8$, and $\min \{-\mu, \eta-\mu\}>-2$; then

$$
V^{\lambda+1, \mu, \eta}(k, \alpha) \subset V^{\lambda, \mu, \eta}\left(k, \alpha_{1}\right),
$$

where $\alpha_{1}$ is given by (18).

Proof. To prove the inclusion relationship, we observe (in view of Theorem 9) that

$$
\begin{aligned}
f(z) \in V^{\lambda+1, \mu, \eta}(k, \alpha) & \Longleftrightarrow z f^{\prime}(z) \in R^{\lambda+1, \mu, \eta}(k, \alpha) \\
& \Longrightarrow z f^{\prime}(z) \in R^{\lambda, \mu, \eta}\left(k, \alpha_{1}\right) \\
& \Longleftrightarrow f(z) \in V^{\lambda, \mu, \eta}\left(k, \alpha_{1}\right),
\end{aligned}
$$

which establishes Theorem 10.

Theorem 11. Let $f \in \mathbb{A}, 0 \leq \alpha, \beta<1, \eta-\lambda>1 / 8$, and $\min \{-\mu, \eta-\mu\}>-2$; then

$$
T^{\lambda+1, \mu, \eta}(k, \beta, \alpha) \subset T^{\lambda, \mu, \eta}\left(k, \beta_{1}, \alpha_{1}\right),
$$

where $\alpha_{1}$ is given by (18) and $\beta<\beta_{1}<1$ is defined in the proof. Proof. Let $f(z) \in T^{\lambda+1, \mu, \eta}(k, \beta, \alpha)$. Then there exists $g(z) \in$ $R^{\lambda+1, \mu, \eta}(2, \alpha)$ such that

$$
\frac{z\left(\mathcal{S}_{0, z}^{\lambda+1, \mu, \eta} f(z)\right)^{\prime}}{\mathcal{S}_{0, z}^{\lambda+1, \mu, \eta} g(z)} \in P_{k}(\beta) \quad(z \in \mathbb{U}, 0 \leq \beta<1) .
$$

Since $R^{\lambda+1, \mu, \eta}(2, \alpha) \subset R^{\lambda, \mu, \eta}\left(2, \alpha_{1}\right)$, therefore we have

$$
\begin{aligned}
\frac{z\left(\delta_{0, z}^{\lambda, \mu, \eta} g(z)\right)^{\prime}}{\delta_{0, z}^{\lambda, \mu, \eta} g(z)} & =\left(1-\alpha_{1}\right) q(z)+\alpha_{1} \\
& =\left(1-\alpha_{1}\right)\left(q_{1}+\iota q_{2}\right)+\alpha_{1},
\end{aligned}
$$

where $q(z) \in P$. Next consider,

$$
\frac{z\left(\delta_{0, z}^{\lambda, \mu, \eta} f(z)\right)^{\prime}}{\delta_{0, z}^{\lambda, \mu, \eta} g(z)}=\left(1-\beta_{1}\right) h(z)+\beta_{1}
$$

and proceed on the same technique of Theorem 9 (see also [5]); we obtain $f(z) \in T^{\lambda, \mu, \eta}\left(k, \beta_{1}, \alpha_{1}\right)$, where

$$
\begin{aligned}
\beta_{1}= & \left(2 \beta\left[\left(\left(1-\alpha_{1}\right) q_{1}+\alpha_{1}+\eta-\lambda\right)^{2}+\left(1-\alpha_{1}\right)^{2} q_{2}^{2}\right]\right. \\
+ & {\left.\left[\left(1-\alpha_{1}\right) q_{1}+\alpha_{1}+\eta-\lambda\right]\right) } \\
\times & \left(2\left[\left(\left(1-\alpha_{1}\right) q_{1}+\alpha_{1}+\eta-\lambda\right)^{2}+\left(1-\alpha_{1}\right)^{2} q_{2}^{2}\right]\right. \\
& \left.+\left[\left(1-\alpha_{1}\right) q_{1}+\alpha_{1}+\eta-\lambda\right]\right)^{-1} .
\end{aligned}
$$

In view of relation (7), an application of Theorem 11 is obtained as follows.

Theorem 12. Let $f \in \mathbb{A}, 0 \leq \alpha, \beta<1, \eta-\lambda>1 / 8$, and $\min \{-\mu, \eta-\mu\}>-2$; then

$$
T_{*}^{\lambda+1, \mu, \eta}(k, \beta, \alpha) \subset T_{*}^{\lambda, \mu, \eta}\left(k, \beta_{1}, \alpha_{1}\right),
$$

where $\beta_{1}$ and $\alpha_{1}$ are as in Theorem 11.

Lemma 13 (see [19]). Let $p(z)$ be analytic in $\mathbb{U}$ with $p(0)=1$ and $\mathbb{R} p(z)>0, z \in \mathbb{U}$. Then, for $s>0$ and $\eta_{1} \neq-1$ (complex),

$$
\mathbb{R}\left(p(z)+\frac{s z p^{\prime}(z)}{p(z)+\eta_{1}}\right)>0, \quad \text { for }|z|<r_{0}
$$

where $r_{0}$ is given by $r_{0}=\left|1+\eta_{1}\right| / \sqrt{m+\left(m^{2}-\left|\eta_{1}^{2}-1\right|\right)^{1 / 2}}$ and $m=2(s+1)^{2}+\left|\eta_{1}\right|^{2}-1$, and this result is the best possible.

Theorem 14. Let $z \in \mathbb{U}, f \in R^{\lambda, \mu, \eta}(k, 0)$. Then $f \in$ $R^{\lambda+1, \mu, \eta}(k, 0)$ for $|z|<r_{0}$, where

$$
r_{0}=\frac{\eta-\lambda+1}{\sqrt{m+\sqrt{m^{2}-\left|(\eta-\lambda)^{2}-1\right|}}}, \quad m=7+(\eta-\lambda)^{2} .
$$

This radius is exact.

Proof. Let

$$
\frac{z\left(\delta_{0, z}^{\lambda, \mu, \eta} f(z)\right)^{\prime}}{\mathcal{S}_{0, z}^{\lambda, \mu, \eta} f(z)}=p(z)=\left(\frac{k}{4}+\frac{1}{2}\right) p_{1}(z)-\left(\frac{k}{4}-\frac{1}{2}\right) p_{2}(z),
$$


where $p \in P_{k}$ and $p_{1}, p_{2} \in P$ in $\mathbb{U}$. Using a similar argument as in Theorem 9 , we obtain

$$
\begin{aligned}
\frac{z\left(\delta_{0, z}^{\lambda+1, \mu, \eta} f(z)\right)^{\prime}}{\delta_{0, z}^{\lambda+1, \mu, \eta} f(z)}= & p(z)+\frac{z p^{\prime}(z)}{\eta-\lambda+p(z)} \\
= & \left(\frac{k}{4}+\frac{1}{2}\right)\left(p_{1}(z)+\frac{z p_{1}^{\prime}(z)}{p_{1}(z)+\eta-\lambda}\right) \\
& -\left(\frac{k}{4}-\frac{1}{2}\right)\left(p_{2}(z)+\frac{z p_{2}^{\prime}(z)}{p_{2}(z)+\eta-\lambda}\right) .
\end{aligned}
$$

Applying Lemma 13, we get

$$
\mathbb{R}\left(p_{i}(z)+\frac{z p_{i}^{\prime}(z)}{p_{i}(z)+\eta-\lambda}\right)>0 \text { for }|z|<r_{0},
$$

where $r_{0}$ is given by (37). This completes our proof.

Lemma 15 (see [20]). Let $p$ be analytic in $\mathbb{E}$ with $p(0)=1$, and $\delta$ is a complex number satisfying $\mathbb{R} \delta \geq 0,(\delta \neq 0)$; then $\mathbb{R}\left[p(z)+\delta z p^{\prime}(z)\right]>\beta,(0 \leq \beta<1)$ implies $\mathbb{R} p(z)>\beta+(1-$ $\beta)(2 \gamma-1)$, where $\gamma$ is given by

$$
\gamma=\int_{0}^{1}\left(1+t^{\mathbb{R} \delta}\right)^{-1} d t
$$

which is an increasing function of $\mathbb{R} \delta$ and $1 / 2 \leq \gamma \leq 1$. The estimate is sharp in the sense that bound cannot be improved.

Theorem 16. Let $\min \{\eta-\lambda-1,-\mu, \eta-\mu\}>-2$. Then

$$
P_{k}^{\prime}(\lambda+1, \mu, \eta, \alpha) \subset P_{k}^{\prime}(\lambda, \mu, \eta, \alpha+(1-\alpha)(2 \gamma-1)),
$$

where

$$
\gamma=\int_{0}^{1}\left(1+t^{1 /(\eta-\lambda+1)}\right)^{-1} d t
$$

which is an increasing function of $1 /(\eta-\lambda+1)$ and $1 / 2 \leq \gamma<1$.

Proof. Let $f(z) \in P_{k}^{\prime}(\lambda+1, \mu, \eta, \alpha)$

$$
\left(\mathcal{S}_{0, z}^{\lambda, \mu, \eta} f(z)\right)^{\prime}=H(z)=\left(\frac{k}{4}+\frac{1}{2}\right) h_{1}(z)-\left(\frac{k}{4}-\frac{1}{2}\right) h_{2}(z),
$$

where $H(z)$ is analytic and $H(0)=1$ in $\mathbb{U}$. Identity (16) gives us

$$
\begin{aligned}
\left(\mathcal{S}_{0, z}^{\lambda+1, \mu, \eta} f(z)\right)^{\prime}= & H(z)+\frac{z H^{\prime}(z)}{\eta-\lambda+1} \\
= & \left(\frac{k}{4}+\frac{1}{2}\right)\left(h_{1}(z)+\frac{z h_{1}^{\prime}(z)}{\eta-\lambda+1}\right) \\
& -\left(\frac{k}{4}-\frac{1}{2}\right)\left(h_{2}(z)+\frac{z h_{2}^{\prime}(z)}{\eta-\lambda+1}\right) .
\end{aligned}
$$

This implies that

$$
\mathbb{R}\left(h_{i}(z)+\frac{z h_{i}^{\prime}(z)}{\eta-\lambda+1}\right)>\alpha, \quad i=1,2 .
$$

Now using Lemma 15, we get the desired result.

Lemma 17 (see [21]). Let $\phi$ be convex and $g$ starlike in $\mathbb{U}$. Then, for $F$ analytic in $\mathbb{U}$ with $F(0)=1,(\Phi * F g) /(\Phi * g)$ is contained in the convex hull of $F(\mathbb{U})$. By convex hull of a set $X$, one means the intersection of all convex sets that contain $X$.

Theorem 18. Let $\phi$ be a convex function and $f \in R^{\lambda, \mu, \eta}(2, \alpha)$. Then $\phi * f \in R^{\lambda, \mu, \eta}(2, \alpha)$.

Proof. Let $G=\phi * f$, and let

$$
\begin{aligned}
& \phi(z)=z+\sum_{n=2}^{\infty} b_{n} z^{n}, \\
& f(z)=z+\sum_{n=2}^{\infty} a_{n} z^{n} .
\end{aligned}
$$

Then

$$
\begin{aligned}
\mathcal{S}_{0, z}^{\lambda, \mu, \eta} G(z)= & \mathcal{S}_{0, z}^{\lambda, \mu, \eta}\left[z+\sum_{n=2}^{\infty} a_{n} b_{n} z^{n}\right] \\
= & z+\frac{\Gamma(2-\mu) \Gamma(2+\eta-\lambda)}{\Gamma(2-\mu+\eta)} \\
& \times \sum_{n=2}^{\infty} \frac{\Gamma(n+1) \Gamma(n-\mu+\eta+1)}{\Gamma(n-\mu+1) \Gamma(n+\eta-\lambda+1)} a_{n} b_{n} z^{n} \\
= & \left(\phi * \mathcal{S}_{0, z}^{\lambda, \mu, \eta} f\right)(z) .
\end{aligned}
$$

Also, $f(z) \in R^{\lambda, \mu, \eta}(2, \alpha)$. Therefore, $\mathcal{S}_{0, z}^{\lambda, \mu, \eta} f(z) \in R_{2}(\alpha)=$ $S^{*}(\alpha)$. By logarithmic differentiation of (48), we have

$$
\frac{z\left(\mathcal{S}_{0, z}^{\lambda, \mu, \eta} G(z)\right)^{\prime}}{\mathcal{S}_{0, z}^{\lambda, \mu, \eta} G(z)}=\frac{\phi(z) * F \mathcal{S}_{0, z}^{\lambda, \mu, \eta} f(z)}{\phi(z) * \mathcal{S}_{0, z}^{\lambda, \mu, \eta} f(z)},
$$

where

$$
F(z)=\frac{z\left(\delta_{0, z}^{\lambda, \mu, \eta} f(z)\right)^{\prime}}{\delta_{0, z}^{\lambda, \mu, \eta} f(z)}
$$

is analytic in $\mathbb{U}$, and $F(0)=1$. From Lemma 17 , we see that $z\left(\mathcal{S}_{0, z}^{\lambda, \mu, \eta} G(z)\right)^{\prime} / \mathcal{S}_{0, z}^{\lambda, \mu, \eta} G(z)$ is contained in the convex hull of $F(\mathbb{U})$. Since $z\left(\mathcal{S}_{0, z}^{\lambda, \mu, \eta} G(z)\right)^{\prime} / \mathcal{S}_{0, z}^{\lambda, \mu, \eta} G(z)$ is analytic in $\mathbb{U}$ and $F(\mathbb{U}) \subset \Omega=\left\{W:\left(z\left(\mathcal{S}_{0, z}^{\lambda, \mu, \eta} W(z)\right)^{\prime}\right) /\left(\mathcal{S}_{0, z}^{\lambda, \mu, \eta} W(z)\right) \in P_{2}(\alpha)\right\}$, then $z\left(\mathcal{S}_{0, z}^{\lambda, \mu, \eta} G(z)\right)^{\prime} / \mathcal{S}_{0, z}^{\lambda, \mu, \eta} G(z)$ lies in $\Omega$. This implies that $G=\phi * f \in R^{\lambda, \mu, \eta}(2, \alpha)$.

This is application of Theorem 18. 
Theorem 19. The class $R^{\lambda, \mu, \eta}(2, \alpha)$ is invariant under the following differintegral operators. That is if $f \in R^{\lambda, \mu, \eta}(2, \alpha)$, then so does $f_{i}$, where $f_{i}$ are given to us:

(i) $f_{1}(z)=\int_{0}^{z}(f(t) / t) d t$,

(ii) $f_{2}(z)=(2 / z) \int_{0}^{z} f(t) d t$,

(iii) $f_{3}(z)=\int_{0}^{z}((f(t)-f(x t)) /(t-x t)) d t,|x| \leq 1, x \neq 1$,

(iv) $f_{4}(z)=\left((1+c) / z^{c}\right) \int_{0}^{z} t^{c-1} f(t) d t, \mathbb{R e}(c)>0$.

The proof immediately follows from Theorem 18. Since we can write $f_{i}=f * \phi_{i}$ with

$$
\begin{gathered}
\phi_{1}(z)=-\log (1-z), \\
\phi_{2}(z)=-2\left[\frac{z+\log (1-z)}{z}\right], \\
\phi_{3}(z)=\frac{1}{1-x} \log \left(\frac{1-x z}{1-z}\right), \\
\phi_{4}(z)=\sum_{m=1}^{\infty} \frac{1+c}{m+c} z^{m} \operatorname{Re} c>0,
\end{gathered}
$$

each $\phi_{i}$ is convex for $i=1,2,3$, and 4 .

\section{Acknowledgment}

The authors would like to express their thanks to the referee for the careful reading and suggestions made for the improvement of the paper.

\section{References}

[1] K. S. Padmanabhan and R. Parvatham, "Properties of a class of functions with bounded boundary rotation," Annales Polonici Mathematici, vol. 31, no. 3, pp. 311-323, 1975.

[2] B. Pinchuk, "Functions of bounded boundary rotation," Israel Journal of Mathematics, vol. 10, pp. 6-16, 1971.

[3] K. I. Noor, "On analytic functions related to certain family of integral operators," Journal of Inequalities in Pure and Applied Mathematics, vol. 7, no. 2, article 69, pp. 1-6, 2006.

[4] K. I. Noor, M. A. Noor, and E. Al-said, "Analytic functions defined by a certain integral operator," International Journal of the Physical Sciences, vol. 6, no. 8, pp. 1876-1881, 2011.

[5] J. K. Prajapat, "Inclusion properties for certain classes of analytic functions involving a family of fractional integral operators," Fractional Calculus \& Applied Analysis, vol. 11, no. 1, pp. 27-34, 2008.

[6] K. I. Noor, "On quasi convex functions and related topics," International Journal of Mathematics and Mathematical Sciences, vol. 10, no. 2, pp. 241-258, 1987.

[7] K. I. Noor, On close-to-convex and related functions [Ph.D. thesis], University of Wales, Wales, UK, 1972.

[8] H. M. Srivastava, M. Saigo, and S. Owa, "A class of distortion theorems involving certain operators of fractional calculus," Journal of Mathematical Analysis and Applications, vol. 131, no. 2, pp. 412-420, 1988.
[9] R. K. Raina and T. S. Nahar, "Certain subclasses of analytic $p$ valent functions with negative coefficients," Informatica, vol. 9 , no. 4, pp. 469-478, 1998.

[10] J. K. Prajapat, R. K. Raina, and H. M. Srivastava, "Some inclusion properties for certain subclasses of strongly starlike and strongly convex functions involving a family of fractional integral operators," Integral Transforms and Special Functions, vol. 18, no. 9-10, pp. 639-651, 2007.

[11] R. K. Raina and H. M. Srivastava, "A certain subclass of analytic functions associated with operators of fractional calculus," Computers \& Mathematics with Applications, vol. 32, no. 7, pp. 13-19, 1996.

[12] S. P. Goyal and J. K. Prajapat, "New class of analytic functions involving certain fractional differ-integral operators," in Proceedings of the 4th Annual Conference of the Society for Special Functions and Their Applications (SSFA), vol. 4, pp. 27-35, 2003.

[13] S. P. Goyal and J. K. Prajapat, "A new class of analytic p-valent functions with negative coefficients and fractional calculus operators," Tamsui Oxford Journal of Mathematical Sciences, vol. 20, no. 2, pp. 175-186, 2004.

[14] S. Kant, "Some subordination results involving generalized fractional differintegral operators," International Journal of Mathematical Sciences and Engineering Applications, vol. 6, no. 2, pp. 1-15, 2012.

[15] J. K. Prajapat and M. K. Aouf, "Majorization problem for certain class of $p$-valently analytic function defined by generalized fractional differintegral operator," Computers \& Mathematics with Applications, vol. 63, no. 1, pp. 42-47, 2012.

[16] I. B. Jung, Y. C. Kim, and H. M. Srivastava, "The Hardy space of analytic functions associated with certain one-parameter families of integral operators," Journal of Mathematical Analysis and Applications, vol. 176, no. 1, pp. 138-147, 1993.

[17] S. Miller, "Differential inequalities and Carathéodory functions," Bulletin of the American Mathematical Society, vol. 81, pp. 79-81, 1975.

[18] K. I. Noor, "On some differential operators for certain classes of analytic functions," Journal of Mathematical Inequalities, vol. 2, no. 1, pp. 129-137, 2008.

[19] S. Ruscheweyh and V. Singh, "On certain extremal problems for functions with positive real part," Proceedings of the American Mathematical Society, vol. 61, no. 2, pp. 329-334, 1976.

[20] R. Singh and S. Singh, "Convolution properties of a class of starlike functions," Proceedings of the American Mathematical Society, vol. 106, no. 1, pp. 145-152, 1989.

[21] St. Ruscheweyh and T. Sheil-Small, "Hadamard products of Schlicht functions and the Pólya-Schoenberg conjecture," Commentarii Mathematici Helvetici, vol. 48, pp. 119-135, 1973. 


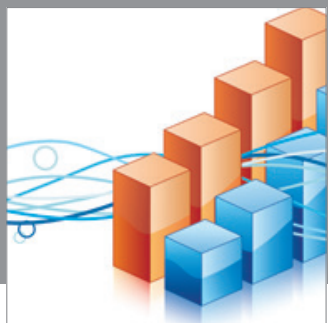

Advances in

Operations Research

mansans

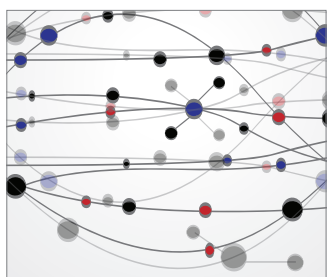

The Scientific World Journal
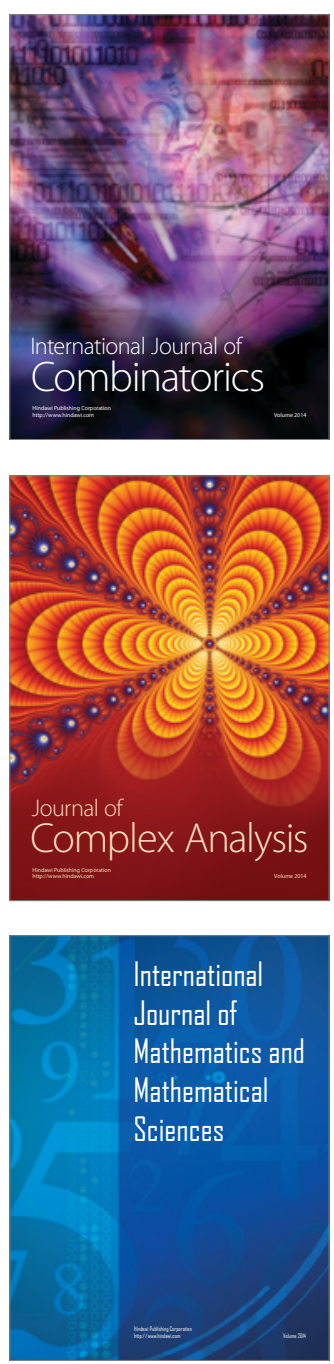
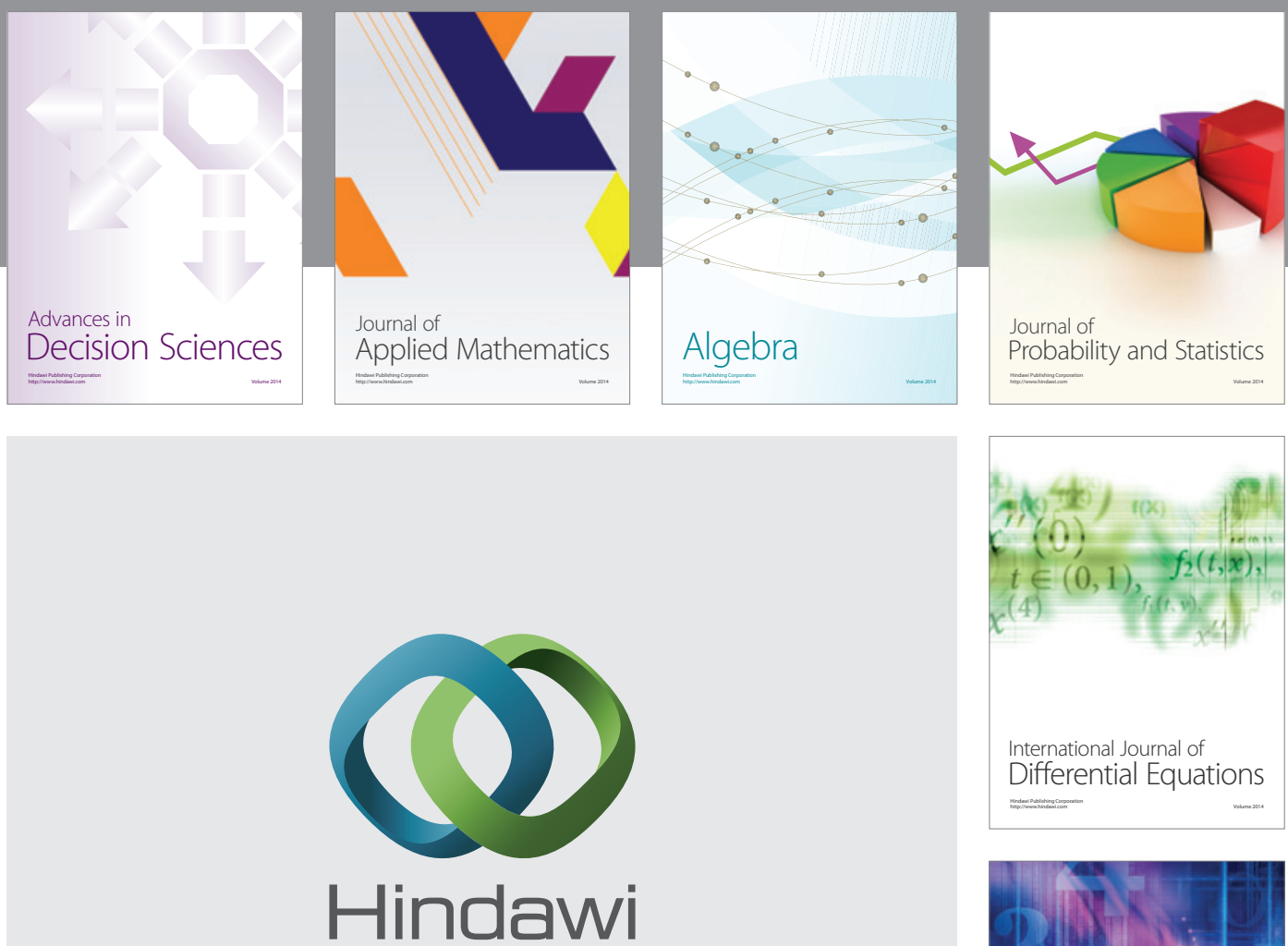

Submit your manuscripts at http://www.hindawi.com
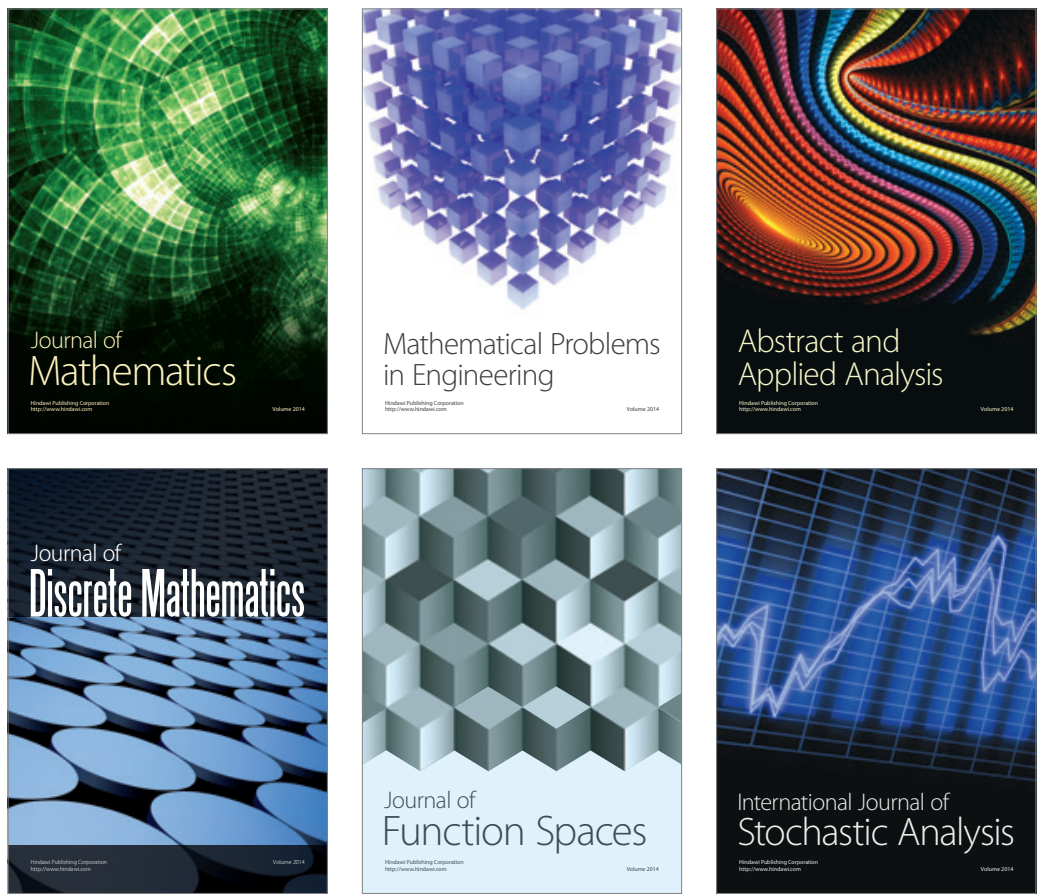

Journal of

Function Spaces

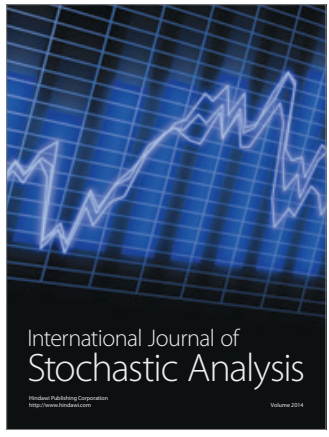

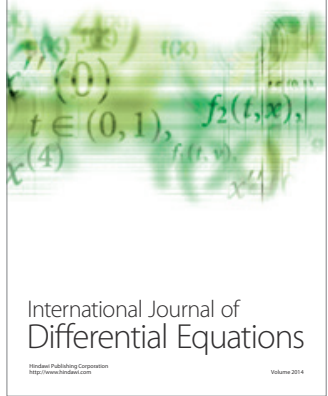
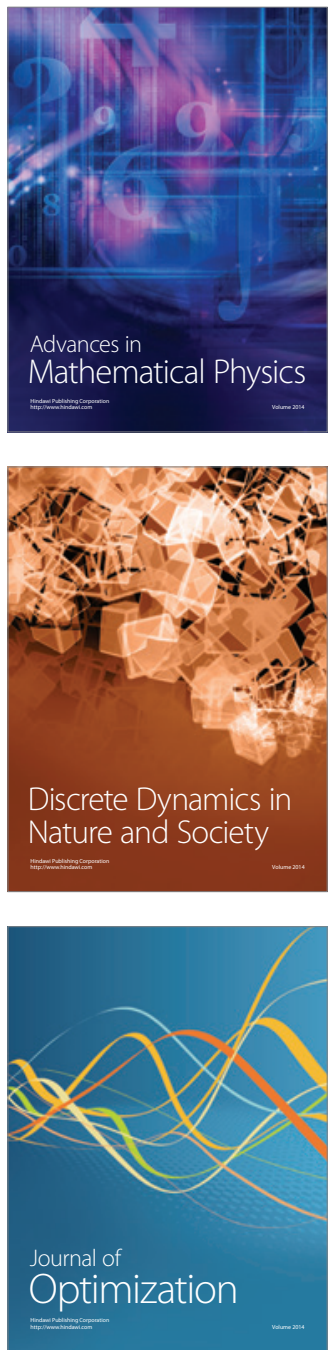